\title{
Improving Physical-Layer Security for Full-duplex Radio aided Two-Way Relay Networks
}

\author{
Shenghua Zhai ${ }^{12^{*}}$, Jianping An ${ }^{1}$ \\ ${ }^{1}$ School of Information and Electronics, Beijing Institute of Technology \\ Beijing 100081,China \\ ${ }^{2}$ CAST-Xi'an Institute of Space Radio Technology \\ Xi' an 710100,China \\ [e-mail: zhaish504@163.com, an@bit.edu.cn] \\ *Corresponding author: Shenghua Zhai
}

Received April 25, 2019; revised June 23, 2019; accepted July 8, 2019; published February 29, 2020

\begin{abstract}
The power allocation optimization problem is investigated for improving the physical-layer security in two-way relaying networks, where a full-duplex relay based half-jamming protocol (HJP-FDR) is considered. Specially, by introducing a power splitter factor, HJP-FDR divides the relay's power into two parts: one for forwarding the sources' signals, the other for jamming. An optimization problem for power split factor is first developed, which is proved to be concave and closed-form solution is achieved. Moreover, we formulate a power allocation problem to determine the sources' power subject to the total power constraint. Applying the achieved closed-form solutions to the above-mentioned problems, a two-stage strategy is proposed to implement the overall power allocation. Simulation results highlight the effectiveness of our proposed algorithm and indicate the necessity of optimal power allocation.
\end{abstract}

Keywords: Full-duplex, Two-way relay network, Physical-layer security, Power allocation 


\section{Introduction}

Cooperative relays have recently been the subject of great interests among the research community owing to their abilities to achieve a broader coverage of wireless networks and obtain the diversity gain [1][2]. Depending on whether the relay broadcasts the two sources' overlapped signals, there are two types of cooperative relaying, i.e., one-way relay (OWR) [3] and two-way relay (TWR) [4]. Due to the half-duplex (HD) [5] mode, one bi-directional communication process costs four time slots for OWR. Fortunately, TWR is able to reduce the process cost to two time slots by allowing the two-way communication be performed in the same channel, simutaneously. Therefore, TWR can greatly improve the spectral efficiency [6]. Particularly, TWR based on amplify-and-forward (TWR-AF) protocol has attracted prominent attentions in many aspects due to its low-complexity in many ascepts, such as relay selection [7], performance evaluation [2] [8], and resource allocation [9].

However, wireless communication systems are also open to potential eavesdroppers because of the broadcasting characteristics of wireless channels. Therefore, physical-layer security (PLS) has become one of key issues for wireless communicatons and attracted great attentions [10][11]. Compared with traditional higher layers' cryptographic methods, PLS technologies protect the communications against eavesdroppers through the untilization of the physical characteristics of wireless channels. In [12], a joint relay and jammer selection in TWR-AF was investigated, where two or three intermediate nodes were selected to enhance PLS. It has been shown that cooperative jamming is able to achieve considerable performance gains compared with the non-jamming schemes. Following the study, [13] proposed a Stackelberg-game-based power allocation scheme to deal with the tradeoff between the friendly jammers and the source nodes. However, the jamming process may also degrade the signal-to-noise ratio (SNR) of legitimate nodes. To overcome this problem, the authors in [14][15] have indicated that: the information leakage mainly happened in phase one and the jamming process in phase two was not necessary. Particularly, to improve the system secrecy performance, [15] adopted a jammer to interfere with the eavesdroppers in the first time slot. In this way, the impact of jamming process on legitimate links is reduced.

To further reduce the negative impact of jamming process, full-duplex radio (FDR) has recently been introduced. FD allows the node to transmit and receive signals asynchronously over the same frequency band [17]-[21]. In [16], a half-jamming protocol based on FDR (HJP-FDR) was developed. Specially, instead of adopting additional jammer, the relay was responsible for the duties of both receiving and jamming by operating in FD mode in the first time slot. The relay can perfectly remove its own jamming signal by using the methods in [18], [22] during the second time slot. Therefore, the negative impact of jamming process can be eliminated. However, only equal power allocation was presented in [16], thus its performance improvement was heavily limited. Therefore, in this paper, we develop the optimization problem to choose properly the power splitter factor and source power allocation for HJP-FDR in TWR-AF. The contributions of our work can be summarized as follows:

- Power split factor: An optimization problem is developed to choose properly the power splitter factor for jamming. By simplifying the objective function, we have proved the problem is concave and derived its closed-form solution.

- Source power allocation: We formulate a power allocation problem to determine the sources' power subject to total power constraint. By replacing the exact capacity expressions with their upper bounds, the simplification of the objective function is given. Closed-form 
solution is then obtained.

- Two-stage strategy: It has been shown that the solution to the power splitter factor is independent with that of the source power allocation. Therefore, a two-stage strategy is finally presented to implement the joint optimization of source power and power splitter factor.

We organize the rest of our paper as follows. System model is firstly introduced in Section II. The optimal power allocation is developed in Section III. Simulation results and conclusion are given in Section IV and Section V respectively.

\section{System Model}

This paper considers a wireless two-way relay network with four nodes: two sources $A$ and $B$, one relay $R$ and one eavesdropper $E$, as shown in Fig. 1. $R$ helps the communications of $A$ and $B$, while $E$ attempts to intercept the private information. The relay can switch between HD mode and FD mode, while both source nodes operate in HD mode. Particularly, by introducing a power splitter factor $p(0 \leq p \leq 1)$, we divide the relay's power, $P_{R}$, into two parts: $(1-p) P_{R}$ is for forwarding the sources' signals, and $p P_{R}$ is for jamming. The detailed protocol, i.e., HJP-FDR [16], is illustrated by Fig. 1.

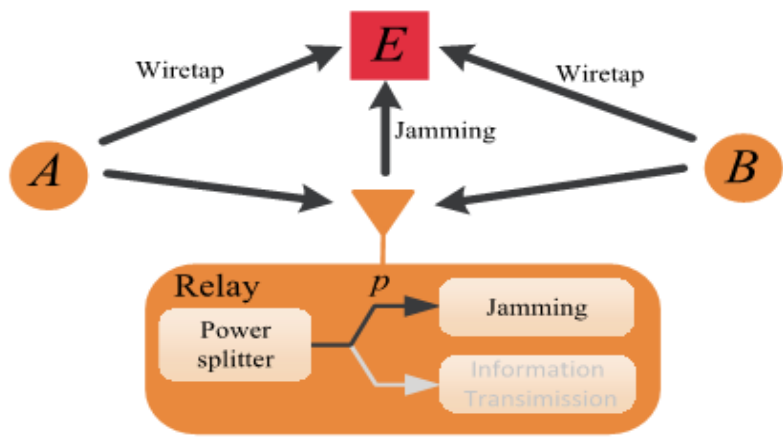

1st time slot: Both $A$ and $B$ transmit their signals to $R$, simultaneously. $R$ operates in FD mode and transmitting jamming while it simultaneously receiving the sources' signals.

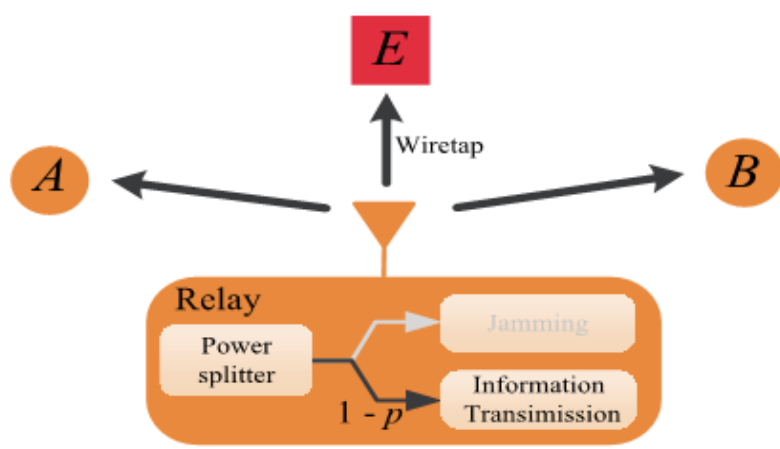

2nd time slot: $R$ first removes their self-interference and then operates in HD mode to broadcast the superimposed signal after amplifying it with a variable gain $G$.

Fig. 1. System model and illustration for HJP-FDR protocol. 
Obviously, HJP-FDR does not employ the jamming process in the relay's broadcasting time slot. This is because the information leakage mainly happened in phase one and the jamming process in phase two was not necessary [14] [15]. Particularly, the jamming process in HJP-FDR has no impact on the two sources, since $R$ is able to eliminate its self-interference following the schemes in [18] [23]. Moreover, HJP-FDR does not require additional jamming node, which has greatly reduced the complexity of the whole system.

Below, we first directly present the performance of the legitimate links and then derive in detail the performance of the wiretapped links.

The instantaneous SNRs for links $B \rightarrow A$ and $A \rightarrow B$ can be given by [14]

$$
\begin{aligned}
& \Upsilon_{A}^{D}=\frac{(1-p) P_{R} P_{B} g_{A R} g_{B R}}{\left((1-p) P_{R}+P_{A}\right) g_{A R}+P_{B} g_{B R}} \\
& \Upsilon_{B}^{D}=\frac{(1-p) P_{R} P_{A} g_{A R} g_{B R}}{\left((1-p) P_{R}+P_{B}\right) g_{B R}+P_{A} g_{A R}}
\end{aligned}
$$

where $P_{A}, P_{B}$ denote respectively the transmit power of $A, B . g_{A R}=\left|h_{A R}\right|^{2} / N_{0}$ and $g_{B R}=\left|h_{B R}\right|^{2} / N_{0}$, in which $h_{A R}$ and $h_{B R}$ denote the channel gain of links $A-R$ and $B-R$ respectively, and $N_{0}$ is the power spectral density of the noise. Then, the capacities of the desired channels are given by

$$
\begin{aligned}
& C_{A}^{D}=\frac{1}{2} \log _{2}\left(1+\Upsilon_{A}^{D}\right), \\
& C_{B}^{D}=\frac{1}{2} \log _{2}\left(1+\Upsilon_{B}^{D}\right) .
\end{aligned}
$$

For the wirtapped links, in phase one, $R$ operates in FD mode and receives the sources' signals with power (1-p) $P_{R}$, while it simultaneously broadcasts the jamming noise to $E$. The received signal at $E$ in phase one can be written as

$$
y_{E R}^{(1)}(t)=\sqrt{P_{A}} h_{A E} s_{A}(t)+\sqrt{P_{B}} h_{B E} s_{B}(t)+\sqrt{p P_{R}} h_{E R} s_{J}(t)+n_{1}(t)
$$

where $s_{A}(t)$ and $s_{B}(t)$ denote respectively the signals transmitted by $A$ and $B$ with unit power. $n_{1}(t) \in C N\left(0, N_{0}\right)$ is the additive white Gaussian noise (AWGN) at $E$, and $s_{J}(t)$ is the jamming noise from $R$. The wiretapped SNRs from $A$ and $B$ in phase one are given by

$$
\begin{gathered}
\Upsilon_{A}^{E(1)}=\frac{P_{B} g_{B E}}{P_{A} g_{A E}+p P_{R} g_{E R}+1}, \\
\Upsilon_{B}^{E(1)}=\frac{P_{A} g_{A E}}{P_{B} g_{B E}+p P_{R} g_{R E}+1} .
\end{gathered}
$$


where $g_{A E}=\left|h_{A E}\right|^{2} / N_{0}, g_{B E}=\left|h_{B E}\right|^{2} / N_{0}, h_{A E}$ and $h_{B E}$ denote respectively the channel gain of links $A-E$ and $B-E$.

In phase two, $R$ first rebuilds and perfectly eliminates its self-interference, then forwards the signal with a variable gain by operating in HD mode. For the sake of mathematical tractability, we use a widely adopted approximation amplifying gain $G=\sqrt{(1-p) P_{R} /\left(P_{A}\left|h_{A R}\right|^{2}+P_{B}\left|h_{B R}\right|^{2}\right)}$ [14], [24] by ignoring the noise statistic at the relay. It has been demonstrated that the relative error of this approximation is low even when SNR $=0 \mathrm{~dB}$ [25]. Thus, the received signal at $E$ is given by

$$
y_{R E}^{(2)}(t)=h_{R E} G\left(\sqrt{P_{A}} h_{A R} s_{A}(t)+\sqrt{P_{B}} h_{B R} s_{B}(t)+n_{2}(t)\right)+n_{3}(t)
$$

where $n_{2}(t) \in C N\left(0, N_{0}\right)$ and $n_{3}(t) \in C N\left(0, N_{0}\right)$ denote respectively the AWGN at $R$ and $E$. Then the wiretapped SNRs from $A$ and $B$ in the phase two can be written as

$$
\begin{aligned}
& \Upsilon_{A}^{E(2)}=\frac{(1-p) P_{R} P_{B} g_{B R} g_{E R}}{(1-p) P_{R} P_{A} g_{E R} g_{A R}+E_{E R}} \\
& \Upsilon_{B}^{E(2)}=\frac{(1-p) P_{R} P_{A} g_{A R} g_{E R}}{(1-p) P_{R} P_{B} g_{E R} g_{B R}+E_{E R}}
\end{aligned}
$$

where $E_{\mathrm{ER}}=(1-p) P_{R} g_{E R}+P_{A} g_{A R}+P_{B} g_{B R}$. Thus, the wiretapped rate from $B$ and $A$ to $E$ can be written as

$$
\begin{aligned}
& C_{A}^{E}=\frac{1}{2} \log _{2}\left(1+\Upsilon_{A}^{E}\right), \\
& C_{B}^{E}=\frac{1}{2} \log _{2}\left(1+\Upsilon_{B}^{E}\right),
\end{aligned}
$$

where $\Upsilon_{i}^{E}=\Upsilon_{i}^{E(1)}+\Upsilon_{i}^{E(2)}$ with $i \in\{A, B\}$.

In summary, the secrecy rates for links $B \rightarrow A$ and $A \rightarrow B$ can be presented as

$$
\begin{aligned}
& C_{A}^{S}=C_{A}^{D}-C_{A}^{E}, \\
& C_{B}^{S}=C_{B}^{D}-C_{B}^{E} .
\end{aligned}
$$

Then, the sum secrecy rate (SSR) can be presented as

$$
C^{S-s u m}=C_{A}^{S}+C_{B}^{S} .
$$




\section{Power Allocation}

\subsection{Power Splitter Factor $p$}

The objective of this subsection is to choose properly the power splitter factor $p$. Specifically, we consider the following problem:

\section{P1: Optimal problem for $p$}

$$
\begin{aligned}
& \max _{p} C^{S-\text { sum }} \\
& \text { s.t. } \quad 0 \leq p \leq 1
\end{aligned}
$$

Since it is difficult to solve the problem in (16) straightforwardly, we suggest a suboptimal approach by approximating $\Upsilon_{A}^{E}$ and $\Upsilon_{B}^{E}$ as:

$$
\begin{aligned}
& \Upsilon_{A}^{E} \approx \frac{P_{B} g_{B E}}{P_{A} g_{A E}+p P_{R} g_{E R}}+\frac{P_{B} g_{B R}}{P_{A} g_{A R}} \\
& \Upsilon_{B}^{E} \approx \frac{P_{A} g_{A E}}{P_{B} g_{B E}+p P_{R} g_{E R}}+\frac{P_{A} g_{A R}}{P_{B} g_{B R}}
\end{aligned}
$$

Then the problem can be represented as $\mathbf{P 2}$ :

\section{P2: Suboptimal problem for $p$}

$$
\max _{p} \frac{\frac{(1-p) P_{R} g_{A R} g_{B R}}{\left((1-p) P_{R}+P_{A}\right) g_{A R}+P_{B} g_{B R}}}{\frac{g_{B E}}{P_{A} g_{A E}+p P_{R} g_{E R}}+\frac{g_{B R}}{P_{A} g_{A R}}} \frac{\left((1-p) P_{R}+P_{B}\right) g_{B R}+P_{A} g_{A R}}{\frac{g_{A E}}{P_{B} g_{B E}+p P_{R} g_{E R}}+\frac{g_{A R}}{P_{B} g_{B R}}}
$$

s.t. $\quad 0 \leq p \leq 1$

The objective function of (19) is concave when $0 \leq p \leq 1$. Therefore, there exists a unique global optimal solution for (19). The brief proof is given as follows.

Proof: For simplicity, we use some general symbols, such as $a_{1}-a_{5}, b_{1}-b_{5}$, to represent the unrelated component data in (19). Thus, the objective function of (19) can be simply denoted as

$$
\varphi_{1}(p) \times \varphi_{2}(p)=\frac{\frac{a_{1}}{1+a_{2}(1-p)}}{\frac{1}{a_{3}+a_{4} p}+a_{5}} \times \frac{\frac{b_{1}}{1+b_{2}(1-p)}}{\frac{1}{b_{3}+b_{4} p}+b_{5}}
$$

We then can get what we wanted by analyzing Equ. (20). The second derivative of $\varphi_{1}(p)$ can be given as 


$$
\begin{aligned}
\varphi_{1}^{\prime \prime}(p)= & -\frac{2 a_{1}}{\left(1+a_{5}\left(a_{4} p+a_{3}\right)\right)^{3}\left(1-p+a_{2}\right)^{3}} \times\left(a_{2}\left(a_{4} p+a_{3}\right)^{3} a_{5}^{2}+\left(a_{2}\left(a_{2}+1\right) a_{4}+a_{3}\right)\right. \\
& \left.+\left(3 a_{2}\left(a_{2} / 3+p+1 / 3\right) a_{3} a_{4}+2 a_{2} a_{3}^{2}+\left(a_{2}^{2}+\xi(p) a_{2}+(1-p)^{3}\right) a_{4}^{2}\right) a_{5}\right)
\end{aligned}
$$

where $\xi(p)=3 p^{2}-3 p+2$ is a quadratic polynomial with one variable and it can be easily proved that $\xi(p) \geq 4 / 11>0$. Therefore, $\varphi_{1}^{\prime \prime}(p)<0$ when $0 \leq p \leq 1$. Similarly, we have $\varphi_{2}^{\prime \prime}(p)<0$ when $0 \leq p \leq 1$. As a result, the objective function of (19) is a concave function.

By setting the derivative of the objective function to be zero, we can obtain the optimal solution as follows:

$$
\frac{1}{g_{E R} P_{R}} \operatorname{Root}\left(\omega_{1} p^{4}+\omega_{2} p^{3}+\omega_{3} p^{2}+\omega_{4} p+\omega_{5}\right)
$$

where $\omega_{1}, \omega_{2}, \ldots, \omega_{5}$ are given in (23). Obviously, (22) is a 4th-order polynomial which has only one root within $[0,1]$. The roots of general polynomials of up to the 4th-order have closed-form expressions, e.g., Ferrari's solution and Euler's solution [26].

$$
\left\{\begin{aligned}
\omega_{1}= & 2 g_{A R} g_{B R} \\
\omega_{2}= & 3 P_{A} g_{A R}^{2} g_{B E}+4 g_{B R}\left(P_{A} g_{A E}+P_{B} g_{B E}\right) g_{A R}+3 P_{B} g_{B R}^{2} g_{A E} \\
\omega_{3}= & \left(2 P_{A}^{2} g_{A R} g_{B R}+6 P_{A} P_{B} g_{B R}^{2}\right) g_{A E}^{2}+\left(6 P_{A} P_{B} g_{B E}^{2}-P_{A} P_{R} g_{B E} g_{E R}\right) g_{A R}^{2}+2 P_{B}^{2} g_{A R} g_{B E}^{2} g_{B R} \\
& +\left(2 P_{A}^{2} g_{A R}^{2} g_{B E}+12 P_{A} P_{B} g_{A R} g_{B E} g_{B R}+2 P_{B}^{2} g_{B E} g_{B R}^{2}-P_{B} P_{R} g_{B R}^{2} g_{E R}\right) g_{A E} \\
\omega_{4}= & P_{B} P_{A}\left(3 P_{B} g_{A R}^{2} g_{B E}^{2}+\left(\left(4 P_{A} g_{A R}^{2}+7 P_{B} g_{A R} g_{B R}\right) g_{A E}-2 P_{R} g_{A R}^{2} g_{E R}\right) g_{B E}^{2}\right. \\
& \left.+\left(\left(7 P_{A} g_{A R}+4 P_{B} g_{B R}\right) g_{A E}-2 P_{R} g_{A R} g_{E R}\right) g_{A E} g_{B R} g_{B E}+g_{A E}^{2} g_{B R}^{2}\left(3 P_{A} g_{A E}-2 P_{R} g_{E R}\right)\right) \\
\omega_{5}= & 2 P_{B}\left(P_{A} g_{B E}\left(P_{B} g_{B E}-P_{R} g_{E R} / 2\right) g_{A E}^{2}+P_{A} P_{B} g_{A R} g_{A E} g_{B E}^{2}-P_{B} P_{R} g_{A R} g_{E R} g_{B E}^{2} / 2\right) \\
& \cdot\left(g_{A R} g_{B E}+g_{B R} g_{A E}\right) P_{A}
\end{aligned}\right.
$$

\subsection{Source Power Allocation}

In this subsection, a power allocation optimization problem is developed for maximizing SSR subject to total power constraint $P_{T}$. Specifically, we consider the following problem:

\section{P3: Optimal problem for source power}

$$
\begin{array}{ll}
\max _{P_{A}, P_{B}, P_{R}} & C^{S-\text { sum }} \\
\text { s.t. } & P_{A}, P_{B}, P_{R} \geq 0 \\
& P_{A}+P_{B}+P_{R} \leq P_{T}
\end{array}
$$

Similarly, it is hard to achieve the optimal solution to the problem in (24), directly. We consider the simplification of the objective function by replacing the exact capacity expressions with their upper bounds as:

$$
\Upsilon_{A R}^{D}<(1-p) P_{R} g_{A R}
$$




$$
\begin{gathered}
\Upsilon_{B R}^{D}<(1-p) P_{R} g_{B R}, \\
\Upsilon_{A R}^{E}<\frac{P_{B} g_{B R}}{P_{A} g_{A R}}, \\
\Upsilon_{B R}^{E}<\frac{P_{A} g_{A R}}{P_{B} g_{B R}} .
\end{gathered}
$$

Through large amounts of numerical simulations, we have found that the value of $P_{R}$ is around $P_{T} / 2$ when the problem (24) approach its optimal value. Therefore, to further simplify the problem, we set $P_{R}=P_{T} / 2$. Then, $\mathbf{P 3}$ can be written as $\mathbf{P 4}$.

\section{P4: Suboptimal problem for source power}

$$
\begin{array}{ll}
\max _{P_{A}, P_{B}, P_{R}} \frac{1+(1-p) P_{T} g_{A R} / 2}{1+P_{B} g_{B R} / P_{A} g_{A R}} \cdot \frac{1+(1-p) P_{T} g_{B R} / 2}{1+P_{A} g_{A R} / P_{B} g_{B R}} \\
\text { s.t. } \quad P_{A}, P_{B}, P_{R} \geq 0 \\
& P_{A}+P_{B}+P_{R} \leq P_{T}
\end{array}
$$

The above problem is non-convex due to the non-convex objective function. However, we still will be able to find the solution to this problem by resorting to the KKT conditions. The Lagrangian function of $\mathbf{P 4}$ is presented as follows:

$$
\begin{aligned}
& \zeta\left(P_{A}, P_{B}, \lambda_{1}, \lambda_{2}, \lambda_{3}\right) \\
& =\frac{1+(1-p) P_{T} g_{A R} / 2}{1+P_{B} g_{B R} / P_{A} g_{A R}} \cdot \frac{1+(1-p) P_{T} g_{B R} / 2}{1+P_{A} g_{A R} / P_{B} g_{B R}}-\lambda_{1} P_{A}-\lambda_{2} P_{B}+\lambda_{3}\left(P_{A}+P_{B}-P_{T} / 2\right) .
\end{aligned}
$$

The solution to $\mathbf{P} \mathbf{4}$ must satisfy the KKT conditions [27] as

$$
\left\{\begin{array}{l}
\frac{\partial \zeta}{\partial P_{A}}=\frac{g_{B R} g_{A R}\left(2+P_{T}(1-p) g_{B R}\right)\left(2-P_{T}(1-p) g_{A R}\right)\left(P_{A} g_{A R}-P_{B} g_{B R}\right) P_{B}}{4\left(P_{A} g_{A R}+P_{B} g_{B R}\right)^{3}}-\lambda_{1}-\lambda_{3}=0 \\
\frac{\partial \zeta}{\partial P_{B}}=\frac{g_{B R} g_{A R}\left(2+P_{T}(1-p) g_{B R}\right)\left(2+P_{T}(1-p) g_{A R}\right)\left(P_{A} g_{A R}-P_{B} g_{B R}\right) P_{A}}{4\left(P_{A} g_{A R}+P_{B} g_{B R}\right)^{3}}-\lambda_{2}-\lambda_{3}=0 . \\
P_{A}+P_{B} \leq P_{T} / 2, \lambda_{3}\left(P_{A}+P_{B}-P_{T} / 2\right)=0 \\
\lambda_{1} P_{A}=0, \lambda_{2} P_{B}=0, P_{A}, P_{B}, \lambda_{1}, \lambda_{2}, \lambda_{3} \geq 0
\end{array} .\right.
$$

Then, a unique optimal solution to $\mathbf{P 4}$ is achieved by solving the KKT conditions in (31):

$$
\begin{aligned}
& P_{A}^{*}=\frac{1}{2} \frac{g_{B R}}{g_{A R}+g_{B R}} P_{T}, \\
& P_{B}^{*}=\frac{1}{2} \frac{g_{A R}}{g_{A R}+g_{B R}} P_{T} .
\end{aligned}
$$

From (33), we can find that the source power allocation is independent from $p$. Therefore, the overall power allocation can be simply implemented by the following two-stage strategy.

- Step I: Allocate the power of the sources and relay according to (33).

- Step II: Based on the power levels of each nodes, figure out the power splitter factor $p$ according to (22).

It is worth noting that our suboptimal design is very close to the optimal counterpart as later shown in simulations, although some approximations are adopted. 


\subsection{Power Allocation without Eavesdropper's CSI}

In this subsection, we consider a more realistic case where the CSI of the eavesdropper is not available. From (32) and (33), we can observe that the source power allocation is independent from CSI of $E$. Therefore, the proposed source power allocation can be adopted directly. However, the power splitter factor, $p$, determined by the CSI of $E$, as shown in (22), which is invalid in this case. Thus, for simplicity, we set $p=0.25$ (as later shown in Fig. 7, $p<=0.25$ in most cases.). Finally, the power allocation for the case without eavesdropper's CSI can be given by:

$$
\left\{\begin{array}{l}
P_{A}^{*}=\frac{1}{2} \frac{g_{B R}}{g_{A R}+g_{B R}} P_{T} \\
P_{B}^{*}=\frac{1}{2} \frac{g_{A R}}{g_{A R}+g_{B R}} P_{T} \\
P_{R}^{*}=P_{T} / 2 \\
\rho=0.25
\end{array}\right.
$$

\section{Numerical Results}

Computer simulations are performed to examine the performance of the proposed power allocation based on the simulation model as shown in Fig. 2. Specifically, the coordinate of node $i$ is denoted by $\left(x_{i}, y_{i}\right)$, where $x_{i}$ denotes horizontal coordinate and $y_{i}$ denotes vertical coordinate. Set $y_{R}=0.5,\left(x_{A}, y_{A}\right)=(-0.5,0)$ and $\left(x_{B}, y_{B}\right)=(0.5,0)$. We consider Rician fading with parameter $K=3$. Let the path loss coefficient $\alpha=3$. For the equal power allocation, $P_{A}$ $=P_{B}=P_{R}=P_{T} / 3, p=0.5$.

SSR is presented in Fig. 3 against $x_{E}$ under the equal power allocation (EPA) [16] and the proposed power allocation with $P_{T}=20 \mathrm{dBm},\left(x_{R}, y_{R}\right)=(0,0.5)$ and $\left(x_{E}, y_{E}\right)=(-0.5 \sim 0.5,0)$. Moreover, to further examine the effectiveness of the proposed algorithm, the optimal SSR under numerical calculation is also presented. The optimal (Numerical) result here is obtained by using the computer to search the optimal set of $\left\{p, P_{R}, P_{A}, P_{B}\right\}$ from the entire feasible region. From Fig. 3, we can observe that the proposed power allocation has similar performance to the optimal counterpart and outperforms EPA. In this scenario, we can observe that SSR falls obviously when $E$ is close to the sources.

Moreover, by setting $P_{T}=20 \mathrm{dBm},\left(x_{R}, y_{R}\right)=(-0.5 \sim 0.5,0.5)$ and $\left(x_{E}, y_{E}\right)=(0,0)$, we compare SSR of the proposed power allocation with EPA against $x_{R}$ in Fig. 4. Similarly, the optimal SSR under numerical calculation is also presented. As shown in Fig. 4, similar to Fig. 3, the proposed power allocation has similar performance as the optimal counterpart and is well ahead of EPA. For EPA, SSR decreases rapidly when $R$ locates closely to the sources. While for the proposed power allocation, SSR is still showing a high level, indicating the necessity of optimal power allocation.

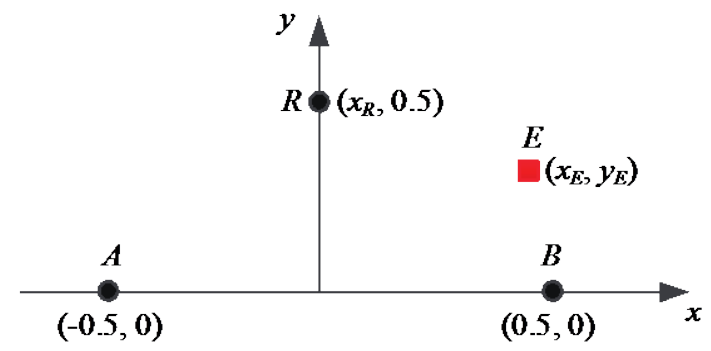

Fig. 2. Simulation model. 


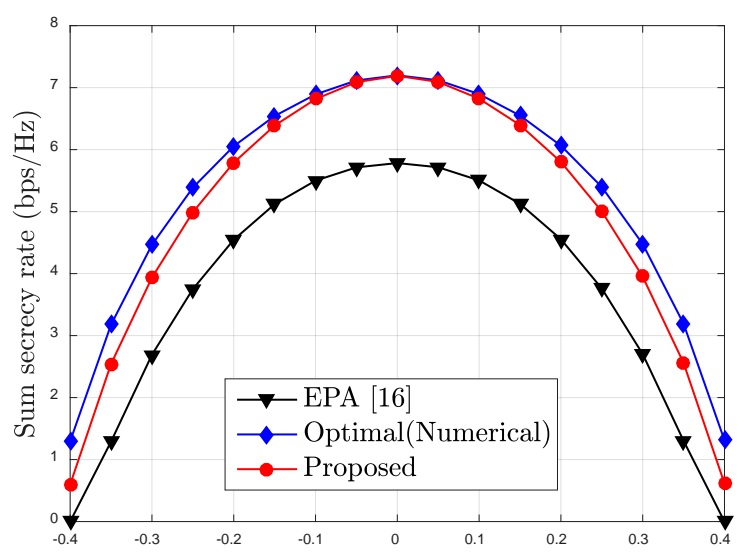

Fig. 3. SSR against $d_{A E}$ with $P_{T}=20 \mathrm{dBm},\left(x_{A}, y_{A}\right)=(-0.5,0),\left(x_{B}, y_{B}\right)=(0.5,0)\left(x_{R}, y_{R}\right)=(0,0.5),\left(x_{E}, y_{E}\right)$ $=(-0.5 \sim 0.5,0)$.

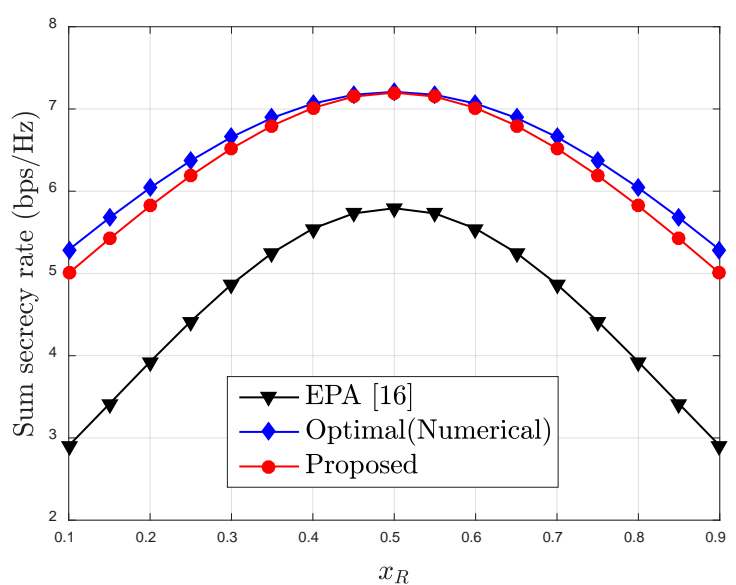

Fig. 4. SSR against $d_{A R}$ with $P_{T}=20 \mathrm{dBm},\left(x_{A}, y_{A}\right)=(-0.5,0),\left(x_{B}, y_{B}\right)=(0.5,0),\left(x_{R}, y_{R}\right)=(-0.5 \sim 0.5,0.5)$, $\left(x_{E}, y_{E}\right)=(0,0)$.

Set $\left(x_{R}, y_{R}\right)=(0,0.5)$ and $\left(x_{E}, y_{E}\right)=(-1 \sim 1,0 \sim 2)$, that is, $E$ traverses the two-dimensional area. SSRs of EPA and the proposed power allocation are plotted in Fig. 5 and Fig. 6 against the location of $E$, where the colors stand for different values of SSR. As can be seen from the two figures, the proposed power allocation has brought considerable performance gain compared with EPA for any locations $E$, highlighting the effectiveness of our design. 


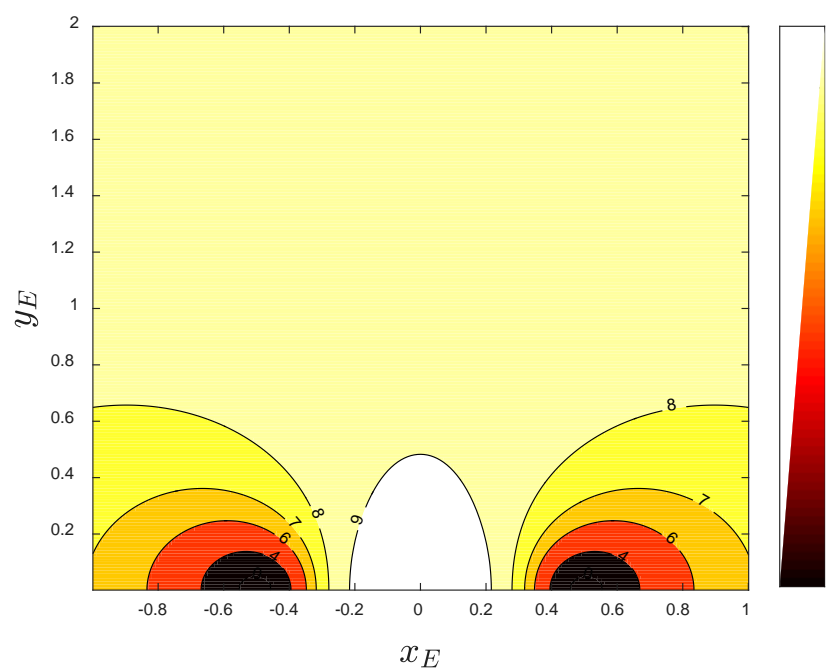

Fig. 5. SSR of EPA [16] against the location of $E$ with $P_{T}=20 \mathrm{dBm},\left(x_{A}, y_{A}\right)=(-0.5,0),\left(x_{B}, y_{B}\right)=(0.5,0)$, $\left(x_{R}, y_{R}\right)=(0,0.5)$ and $\left(x_{E}, y_{E}\right)=(-1 \sim 1,0 \sim 2)$ (different colors stand for different values of SSR).

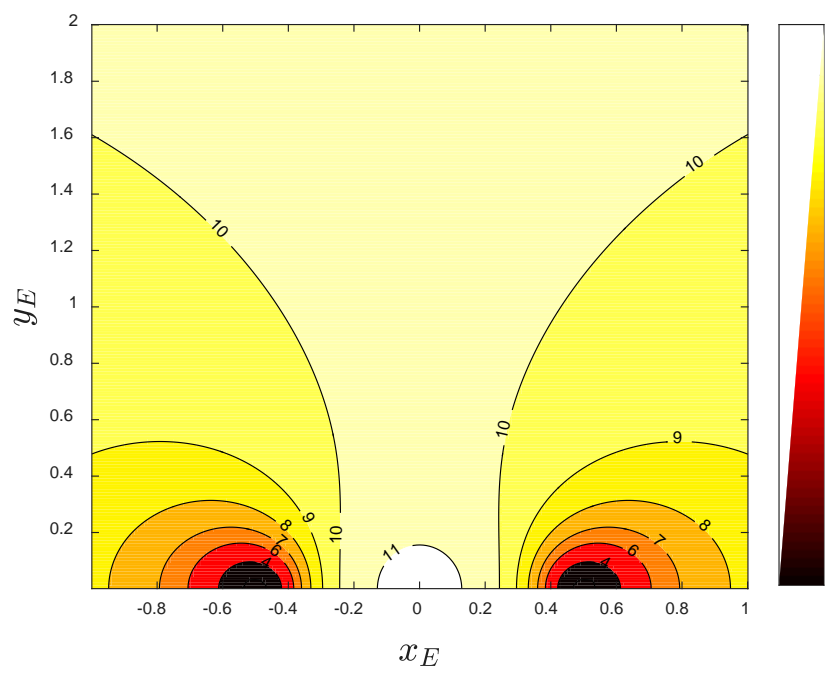

Fig. 6. SSR of the proposed power allocation against the location of $E$ with $P_{T}=20 \mathrm{dBm},\left(x_{A}, y_{A}\right)=(-0.5$, $0),\left(x_{B}, y_{B}\right)=(0.5,0),\left(x_{R}, y_{R}\right)=(0,0.5)$ and $\left(x_{E}, y_{E}\right)=(-1 \sim 1,0 \sim 2)$.

To present the impact of the location of $E$ on the power of jamming, Fig. 7 plots the power splitter factor under the proposed power allocation against the location of $E$ by using the same simulation parameter settings as Fig. 5. In Fig. 7, the colors stand for different values of $p$. As can be observed from Fig. 7, $p$ increases, i.e., more power is allocated for jamming, when $E$ locates closely to the sources. And the jamming power decreases when $E$ locates in the middle between $A$ and $B$, or close to the relay. 


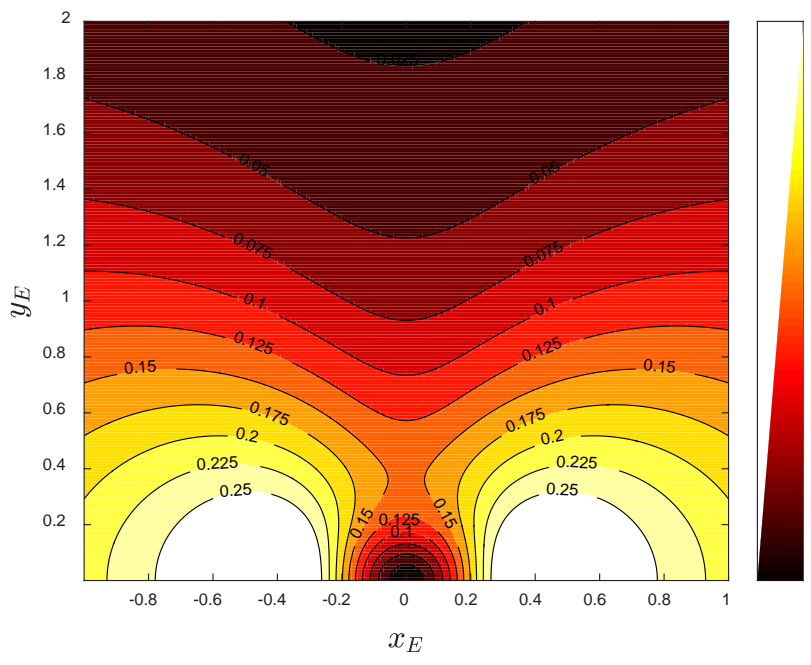

Fig. 7. Power splitter factor under the proposed power allocation against the location of $E$ with $P_{T}=20 \mathrm{dBm},\left(x_{A}, y_{A}\right)=(-0.5,0),\left(x_{B}, y_{B}\right)=(0.5,0),\left(x_{R}, y_{R}\right)=(0,0.5)$ and $\left(x_{E}, y_{E}\right)=(-1 \sim 1,0 \sim 2)$.

To present the impact of $P_{T}$ on SSR, Fig. 8 plots SSR against $P_{T}$ with $\left(x_{A}, y_{A}\right)=(-0.5,0)$, $\left(x_{B}, y_{B}\right)=(0.5,0)\left(x_{R}, y_{R}\right)=(0,0.5),\left(x_{E}, y_{E}\right)=(0,0)$. As shown in Fig. 8, the proposed power allocation is able to achieve non-negative when $P_{T}>7.5 \mathrm{dBm}$, while EPA requires $P_{T}>$ $9.5 \mathrm{dBm}$. Secrecy performance is improved by $2 \mathrm{~dB}$ with the increase of $P_{T}$.

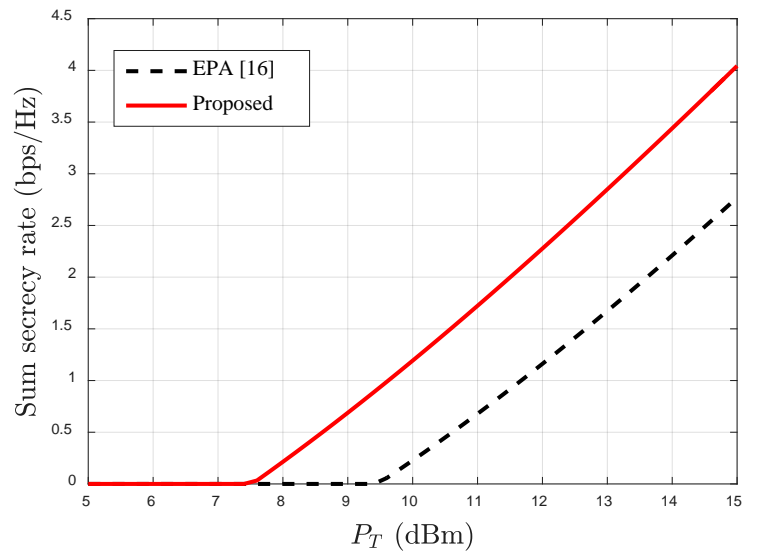

Fig. 8. SSR against $P_{T}$ with $\left(x_{A}, y_{A}\right)=(-0.5,0),\left(x_{B}, y_{B}\right)=(0.5,0)\left(x_{R}, y_{R}\right)=(0,0.5),\left(x_{E}, y_{E}\right)=(0,0)$. 


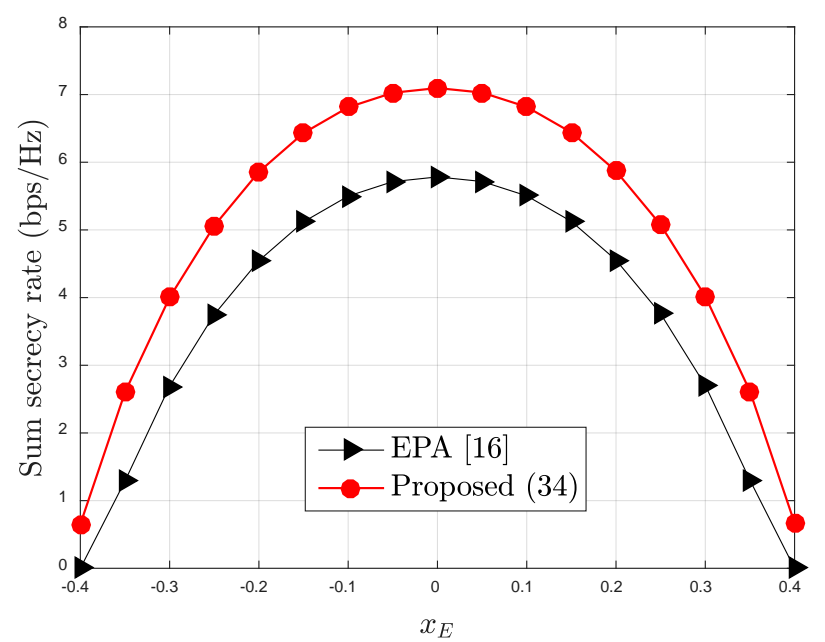

Fig. 9. SSR of EPA and the proposed power allocation in (34) without CSI of $E$.

Finally, we consider the case where CSI of $E$ is not available. The SSR is presented in Fig. 9 against $x_{E}$ under EPA and the proposed power allocation in (34) with $P_{T}=20 \mathrm{dBm},\left(x_{R}, y_{R}\right)=$ $(0,0.5)$ and $\left(x_{E}, y_{E}\right)=(-0.5 \sim 0.5,0)$. From Fig. 9, we can observe that the proposed power allocation still brings considerable performance gain compared with EPA in [16], even though CSI of $E$ is not available.

\section{Conclusion}

A two-stage power allocation strategy for HJP-FDR in TWR-AF is proposed to implement the joint optimization of source power and power splitter factor by using the fact that the solution to the power splitter factor is independent with that to source power allocation, which is derived by developing and solving the optimization problems for power split factor and source power allocation. Moreover, the case without CSI of $E$ has also been taken into consideration. From simulations, we have shown that our proposed algorithm is very close to the optimal counterpart and outperforms equal power allocation, highlighting the necessity of optimal power allocation. It is important to note that the proposed scheme in this paper can also be applied to some specified networks, such as satellite communications where the satellite acts as the relay, multiple relay networks [28], cognitive radio networks [29], and 5G networks [30] [31]. The specific issue of signal synchronization should be addressed when using this method for satellite communications applications.

\section{References}

[1] Y. Shin, and S. J. Baek, "Cooperative buffer-aided relaying using full-duplex relays with flow control," IEEE Trans. on Veh. Technol., vol. 68, no. 2, pp. 1824-1838, Feb. 2019. Article (CrossRef Link)

[2] Y. Li, Y. Z. Li, X. Chu, Y. Ye, and H. Zhang, "Performance analysis of relay selection in cooperative NOMA networks,” IEEE Commun. Letters, vol. 23, no. 4, pp. 760-763, Apr. 2019. Article (CrossRef Link) 
[3] G. Kramer, M. Gastpar, and P. Gupta, "Cooperative strategies and capacity theorems for relay networks,” IEEE Trans. Inf. Theory, vol. 51, no. 9, pp. 3037-3063, Sep. 2005. Article (CrossRef Link)

[4] C. S. Zhang, J. H. Ge, J. Li, Y. Rui, and M. Guizani, “A unified approach for calculating outage performance of two-way AF relaying over fading channels,” IEEE Trans. Veh. Technol., vol. 64, no. 3, pp. 1218-1229, Mar. 2015. Article (CrossRef Link)

[5] B. Rankov and A. Wittneben, "Spectral efficiency protocols for half-duplex fading relay channels," IEEE J. Select. Areas Commun., vol. 25, no. 2, pp. 379-389, Feb. 2007. Article (CrossRef Link)

[6] S. L. Zhang, C. S. Liew and P. P. Lam, "Hot topic: physical-layer network coding," in Proc. of ACM Mobicom, pp.358-365, 2006. Article (CrossRef Link)

[7] K. Singh, M. Lin Ku and J. C. Lin, "Power allocation and relay selection in relay networks: A perturbation-based approach,” IEEE Signal Processing Letters, vol. 24, no. 9, pp. 1328-1332, Jul. 2017. Article (CrossRef Link)

[8] C. S. Zhang, J. H. Ge, J. Li, Y. Rui, and M. Guizani, “A unified approach for calculating outage performance of two-way AF relaying over fading channels,” IEEE Trans. Veh. Technol., vol. 64, no. 3, pp. 1218-1229, Mar. 2015. Article (CrossRef Link)

[9] L. Lu, D. W. He, Q. Q. Xie, G. Y. Li, and X. X. Yu, "Graph-based path selection and power allocation for DF relay-aided transmission,” IEEE Wireless Commun. Letters, vol. 7, no. 1, pp. 138-141, Feb. 2018. Article (CrossRef Link)

[10] J. Zhu, Y. Zou, and B. Zheng, "Physical-layer security and reliability challenges for industrial wireless sensor networks,” IEEE Access, vol. 5, pp. 5313-5320, Apr. 2017. Article (CrossRef Link)

[11] Y. Zou, "Physical-layer security for spectrum sharing systems," IEEE Trans. Wireless Commun., vol. 16, pp. 1319-1329, Feb. 2017. Article (CrossRef Link)

[12] J. Chen, R. Zhang, L. Song, Z. Han, and B. Jiao, "Joint relay and jammer selection for secure two-way relay networks,” IEEE Trans. on Inf. Forensics and Security, vol. 7, no. 1, pp. 310-320, Feb. 2012. Article (CrossRef Link)

[13] F. Jiang, C. Zhu, J. Peng, W. Liu, Z. Zhu, and Y. He, “Joint relay and jammer selection and power control for physical layer security in two-way relay networks with imperfect CSI,” Wireless Pers. Commun., vol. 85, no. 3, pp.841-862, Jun. 2015. Article (CrossRef Link)

[14] C. S. Zhang, J. H. Ge, J. Li, F. K. Gong, and H. Y. Ding, "Complexity-aware relay selection for 5G large-scale secure two-way relay systems,” IEEE Trans. Veh. Technol., vol. 66, no. 6, pp. 5461-5465, Jun. 2017. Article (CrossRef Link)

[15] C. S. Zhang, and J. H. Ge, "Partial jamming for secure two-way relay systems without wiretap information: one >two," Wireless Pers. Commun., vol. 95, no. 4, pp. 4013-4024, Aug. 2017. Article (CrossRef Link)

[16] C. Zhang, J. Ge, Z. Xia, and H. Wang, “A novel half-jamming protocol for secure two-way relay systems using a full-duplex jamming relay,” in Proc. of 2017 3rd IEEE International Conference on Computer and Communications (ICCC), IEEE, pp. 786-790, 2017. Article (CrossRef Link)

[17] Y. Hua, P. Liang, Y. Ma, A. C. Cirik, and Q. Gao, “A method for broadband full-duplex MIMO radio,” IEEE Signal Process. Lett., vol. 19, no. 12, pp. 793-796, Dec. 2012. Article (CrossRef Link)

[18] W. Li, M. Ghogho, B. Chen, and C. Xiong, "Secure communication via sending artificial noise by the receiver: Outage secrecy capacity/region analysis,” IEEE Commun. Lett., vol. 16, no. 10, pp. 1628-1631, Oct. 2012. Article (CrossRef Link)

[19] L. Chen, Q. P. Zhu, W. X. Meng, and Y. B. Hua, "Fast power allocation for secure communication with full-duplex radio,” IEEE Trans. Signal Process., vol. 65, no. 14, pp. 3846-3861, Jul. 2017. Article (CrossRef Link)

[20] G. J. Chen, Y. Gong, P. Xiao, and J. A. Chambers, "Physical layer network security in the full-duplex relay system,” IEEE Trans. on Info. Forensics and Security, vol. 10, no. 3, pp. 574-583, Jan. 2015. Article (CrossRef Link) 
[21] B. Zheng, M. Wen, C.-X. Wang, X. Wang, F. Chen, J. Tang, F. Ji., "Secure NOMA based two-way relay networks using artificial noise and full duplex, ” IEEE J. Select. Areas Commun., vol. 36, no. 7, pp. 1426-1440, Jul. 2018. Article (CrossRef Link)

[22] E. Shafie, A. Sultan, I. Krikidis, N. Al-Dhahir, and R. Hamila, "Achievable rates of buffer-aided full-duplex Gaussian relay channels,” IEEE Access, vol. 5, pp. 23776-23791, Oct. 2017. Article (CrossRef Link)

[23] C. S. Zhang, F. Jia, J. Ge, and F. Gong, "Security-reliability tradeoff for secure relaying systems with full-duplex radio,” IEEE Access, vol. 6, pp. 60862-60868, 2018. Article (CrossRef Link)

[24] P. K. Upadhyay, and S. Prakriya, "Performance of analog network coding with asymmetric traffic requirements,” IEEE Commun. Lett., vol. 15, no. 6, pp. 647-649, Jun. 2011. Article (CrossRef Link)

[25] P. A. Anghel, and M. Kaveh, "Exact symbol error probability of a cooperative network in a Rayleigh-fading environment,” IEEE Trans. Wireless Commun., vol. 3, pp. 1416-1421, Sep. 2004. Article (CrossRef Link)

[26] V. D. Waerden, and B. Leendert, The Galois theory: Equations of the second, third, and fourth degrees, Algebra, 1 (7th ed.), Springer-Verlag, 1991. Article (CrossRef Link)

[27] S. Boyd, and L. Vandenberghe, “Convex optimization,” Cambridge, U.K.: Cambridge Univ. Press, 2004. Article (CrossRef Link)

[28] F. Gong, G. Li , J. Ge ; J. Yuan, "Distributed concatenated recursive Alamouti-circulant STBC for two-way multi-relay networks,” IEEE Trans. on Commun., vol. 64, no.8, pp.3262-3274, Aug. 2016.

[29] X. Gao, P. Wang, D. Niyato, K. Yang, and J. An, “Auction-based time scheduling for backscatter-aided RF-powered cognitive radio networks," IEEE Trans. on Wireless Commun., vol. 18, no. 3, pp. 1684-1697, Mar. 2019. Article (CrossRef Link)

[30] K. Yang, N. Yang, N. Ye, M. Jia, Z. Gao, and R. Fan, "Non-orthogonal multiple access: achieving sustainable future radio access,” IEEE Commun. Magazine, vol. 57, no. 2, pp. 116-121, Feb. 2019. Article (CrossRef Link)

[31] J. An, K. Yang, J. Wu, N. Ye, S. Guo, and Z. Liao, “Achieving sustainable ultra-dense heterogeneous networks for 5G,” IEEE Commun. Magazine, vol. 55, no. 12, pp. 84-90, Dec. 2017. Article (CrossRef Link)

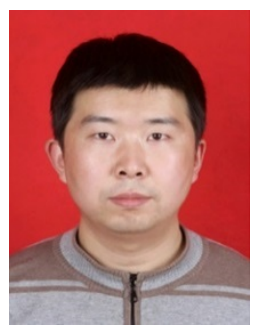

Shenghua Zhai received his Master's degree in college of Information and Communication Engineering from Harbin Engineering University, China, in 2004. He is currently a professor in CAST-Xi'an Institute of Space Radio Technology. His research interests include wireless communication, satellite communication systems, and digital signal processing.

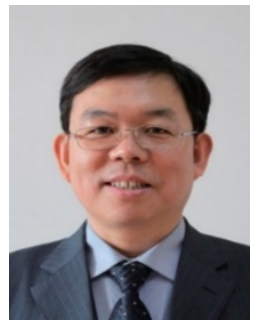

Jianping An received the B.Eng. degree from information Engineering University of the People's Liberation Army, Zhengzhou, China, in 1987 and the Ph.D. degree from Beijing Institute of Technology (BIT) in 1996. From 2010 to 2011 he was a Visiting Professor at University of California, San Diego, USA. He is currently a professor and the Dean of School of Information and Electronics, BIT. His current research interests include signal processing theory and algorithms for communication systems. He has published more than 150 journal and conference articles and holds (or co-holds) 37 patents. He has received various awards for his academic achievements and the resultant industrial influences, including the National Award for Scientific and Technological Progress of China in 1997 and the Excellent Young Teacher Award by the China Ministry of Education in 2000. Since 2010 he has been serving as a Chief Reviewing Expert for the Information Technology Division, National Scientific Foundation of China. 